\title{
ARTICLE
}

Epidemiology

\section{Association between habitual yogurt consumption and newly diagnosed non-alcoholic fatty liver disease}

\author{
Shunming Zhang ${ }^{1} \cdot$ Jingzhu Fu${ }^{1} \cdot$ Qing Zhang ${ }^{2} \cdot \mathrm{Li} \mathrm{Liu}^{2} \cdot \mathrm{Min} \mathrm{Lu}^{3} \cdot \mathrm{Ge}$ Meng ${ }^{1} \cdot$ Zhanxin Yao, ${ }^{1,4} \cdot \mathrm{Hongmei} \mathrm{Wu}^{1}$ • \\ Yang $\mathrm{Xia}^{1} \cdot$ Xue Bao ${ }^{1} \cdot$ Yeqing Gu${ }^{1}$. Shaomei Sun ${ }^{2} \cdot \mathrm{Xing} \mathrm{Wang}^{2} \cdot \mathrm{Ming} \mathrm{Zhou}^{2} \cdot \mathrm{Qiyu} \mathrm{Jia}^{2} \cdot \mathrm{Kun} \mathrm{Song}^{2}$.

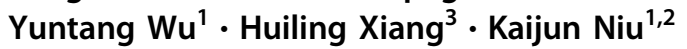

Received: 25 November 2018 / Revised: 20 July 2019 / Accepted: 14 August 2019 / Published online: 2 September 2019

(c) The Author(s), under exclusive licence to Springer Nature Limited 2019

\begin{abstract}
Background/Objectives Many studies have suggested that probiotics may be applied as a therapeutic agent for non-alcoholic fatty liver disease (NAFLD). However, the effects of frequent yogurt consumption (as a natural probiotic source) on NAFLD remain poorly understood. This study was to examine the association of habitual yogurt consumption with newly diagnosed NAFLD in the general adult population.

Subject/Methods Overall, 24,389 adults were included in this cross-sectional study. Yogurt consumption was estimated by using a validated self-administered food frequency questionnaire. NAFLD was diagnosed by abdominal ultrasonography. We used logistic regression models to assess the association between yogurt consumption categories and newly diagnosed NAFLD.

Results The multivariable odds ratios with $95 \%$ confidence interval of newly diagnosed NAFLD were $1.00(0.88,1.14)$ for 1 time/week, $0.91(0.81,1.02)$ for $2-3$ times/week, and $0.86(0.76,0.98)$ for $\geq 4$ times/week $(P$ for trend $=0.01)$, compared with those who consumed $<1$ time/week yogurt. The inverse association was observed in a sensitivity analysis.

Conclusion Higher yogurt consumption was inversely associated with the prevalence of newly diagnosed NAFLD. These results are needed to be confirmed in randomized controlled trials or prospective studies.
\end{abstract}

\section{Introduction}

Non-alcoholic fatty liver disease (NAFLD) is characterized by the accumulation of hepatic fat in the absence of significant alcohol intake. It is a common cause of liver diseases, affecting approximately one-quarter of adults all over the world [1]. About $20 \%$ of adults have NAFLD in the mainland of China, and its prevalence is increasing [2]. It

Kaijun Niu

nkj0809@gmail.com

1 Nutritional Epidemiology Institute and School of Public Health, Tianjin Medical University, Tianjin, China

2 Health Management Centre, Tianjin Medical University General Hospital, Tianjin, China

3 Department of Gastroenterology, Tianjin Third Central Hospital, Tianjin, China

4 Tianjin Institute of Health and Environmental Medicine, Tianjin, China has been reported that NAFLD is associated with an increased risk of cardiovascular disease (CVD) [3, 4]. To date, there is lacking efficacy and safety profiles of pharmacotherapies for NAFLD. Fortunately, lifestyle modification (including diet and exercise) is beneficial for the prevention of NAFLD [5].

Yogurt is a food produced by bacterial fermentation of milk. The consumption of yogurt delivers a large number of probiotics to the gastrointestinal tract [6]. A growing body of evidence shows that probiotics have therapeutic effects for NAFLD [7-10]. Potential mechanisms for these effects include reducing the oxidative and inflammatory liver damage, as well as lowering hepatic triglycerides (TG) and hepatic steatosis $[7,9,11]$. Moreover, probiotics can improve insulin resistance and dyslipidemia, both of which have a prominent role in the pathogenesis of NAFLD [11-13]. Hence, we hypothesized habitual yogurt consumption may protect against the development of NAFLD mainly due to the numerous probiotics contained in yogurt.

To date, two randomized controlled trials (RCTs) have shown that probiotic yogurt consumption could ameliorate 
hepatic steatosis and liver enzyme concentrations in NAFLD patients $[14,15]$. However, there is no study that investigated the association between dietary yogurt intake and NAFLD in a general adult population. Thus, we designed this cross-sectional study to assess whether habitual yogurt consumption is inversely associated with the prevalence of newly diagnosed NAFLD in a large general Chinese adult population.

\section{Methods}

\section{Study design and participants}

The Tianjin Chronic Low-Grade Systemic Inflammation and Health (TCLSIH) Cohort Study is an ongoing large prospective study. This study was launched in 2007. Participants were drawn from health management centers and community management centers during annual health examinations in Tianjin, China. All participants received medical examinations such as abdominal ultrasonography and blood tests. In addition, participants completed questionnaires, regarding their smoking and alcohol drinking status, and provided their disease history since January 2010. All participants provided written informed consent before the study. The study was approved by the Institutional Review Board of Tianjin Medical University. This trial was registered at UMIN Clinical Trials Registry as UMIN000027174.

In this cross-sectional study, we used the baseline data of the TCLSIH Study from May 2013, when participants were administered a detailed lifestyle questionnaire including dietary intake as a standard part of the health checkup process, to December 2016. During the research period, a total of 32,308 subjects received health examinations. Participants with a history of CVD $(n=1694)$ or cancer $(n=$ 346) or other liver diseases (including fatty liver disease, chronic hepatitis B or C, operation on liver, autoimmune liver diseases, or cirrhotic) $(n=2177)$ were excluded. We also excluded those with previously diagnosed NAFLD $(n=3464)$. Moreover, we excluded participants who had missing diet data $(n=238)$. Finally, this cross-sectional sample included 24,389 participants (Fig. 1).

\section{NAFLD diagnosis}

Fatty liver disease was assessed through a standardized abdominal ultrasound examination during annual health examinations. Participants meeting at least two of the following three parameters were diagnosed as fatty liver disease: hyperechogenicity of liver tissue compared to the renal cortex, vascular blurring, and diffuse echogenicity of the liver [16]. Participants with fatty liver disease and no
Total population who had received health examinations $(\mathrm{n}=32,308)$

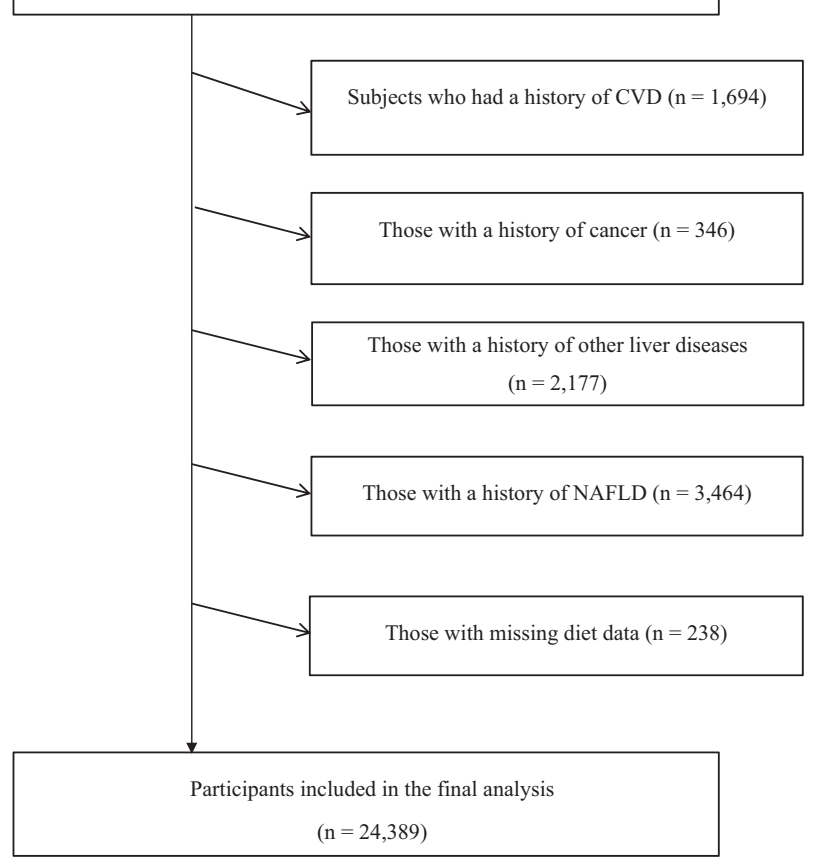

Fig. 1 Participant flow diagram. NAFLD non-alcoholic fatty liver disease, CVD cardiovascular disease

significant alcohol intake ( $<140 \mathrm{~g} /$ week for men and $70 \mathrm{~g} /$ week for women, respectively) were defined as having NAFLD [17]. The newly diagnosed NAFLD was detected in accordance with annual ultrasonic examination results [18].

\section{Assessment of dietary intake}

Data on diet was collected using a validated selfadministered food frequency questionnaire (FFQ). This questionnaire included 100 food items with specified serving sizes, covering 7 response frequency categories ranging from 'almost never eat' to 'twice or more per day' for foods and 8 response frequency categories ranging from 'almost never drink' to 'four or more times per day' for drinks. Daily total energy and nutrient intake were calculated based on the latest available Chinese Food Composition Table [19]. The reproducibility and validity of the FFQ were assessed in a random sample of 150 participants from the TCLSIH Cohort Study [20].

The consumption frequency of dairy products (milk and yogurt) in the previous month was assessed using 7 possible response categories as follows: almost never eat, $<1$ time/ week, 1 time/week, 2-3 times/week, 4-6 times/week, 1 time/day, and $\geq 2$ times/day. For the analyses, intake frequency of yogurt was categorized as (times/week): $<1$ (reference), 1, 2-3, and $\geq 4$. 


\section{Assessment of other variables}

During health examinations, all participants completed a structured questionnaire in regard to their lifestyle factors, including smoking status, alcohol drinking status, and medical history. This questionnaire also collected information on demographics, including birth date (age), sex, household income, educational level, and occupation. Physical activity (PA) in the previous week was determined via the short form of the International Physical Activity Questionnaire [21]. PA was estimated as the total hours of metabolic equivalents per week (MET-h/week).

Body weight, height, and waist circumference (WC) were measured by trained staff using a standardized protocol. Body mass index (BMI) was computed as weight in kilograms divided by the square of height in meters. Blood pressure (BP) was measured following a standardized protocol using TM-2655P (A\&D Company, Ltd., Tokyo, Japan). Participants sat at rest for at least $5 \mathrm{~min}$ in a quiet room before BP measurements, and then kept their upper right arms at heart level with their feet on the ground and their backs supported. Additional measurements were taken if the first two results differed by more than $5 \mathrm{mmHg}$ [22]. At least 1 min elapsed between the measurements. Average values of the two closest readings were used to report BP value of each participant. Participants were defined as having hypertension if they had systolic $\mathrm{BP}(\mathrm{SBP}) \geq 140 \mathrm{mmHg}$ and/or diastolic BP (DBP) $\geq 90 \mathrm{mmHg}$ or a history of hypertension based on the criteria of the JNC 7 [23].

Venous blood samples were collected in siliconized vacuum plastic tubes after an overnight fast. Fasting blood glucose (FBG), total cholesterol (TC), TG, low-density lipoprotein cholesterol (LDL-C), and high-density lipoprotein cholesterol (HDL-C) were measured using appropriate kits on Roche Cobas 8000 modular analyzer (Mannheim, Germany). Participants were considered to have diabetes if they had $\mathrm{FBG} \geq 7.0 \mathrm{mmol} / \mathrm{L}$, or had a self-reported history of diabetes or antidiabetic drug use [24]. Participants were considered to have hyperlipidemia if they met at least one of the following criteria: $\mathrm{TC} \geq 5.17 \mathrm{mmol} / \mathrm{L}$ or $\mathrm{TG} \geq 1.7 \mathrm{mmol} /$ $\mathrm{L}$ or LDL-C $\geq 3.37 \mathrm{mmol} / \mathrm{L}$ or a history of hyperlipidemia or taking antihyperlipidemic drugs. Metabolic syndrome (MS) was determined in accordance with the criteria for China of the American Heart Association and International Diabetes Federation scientific statement of 2009 [25]. White blood cell (WBC) count was determined by an automated hematology analyzer XE-2100 (Sysmex, Kobe, Japan) and expressed as $\times 1000$ cells $/ \mathrm{mm}^{3}[26]$.

\section{Statistical analysis}

To improve the normality of the data, we used the natural logarithm for continuous variables before analysis. Baseline characteristics were presented as geometric means with $95 \%$ confidence interval (CI) for continuous variables and percentages for categorical variables. Baseline participant characteristics between groups were assessed by analysis of variance and logistic regression analysis, as appropriate. Multiple comparisons were corrected using the Bonferroni method. The odds ratios (ORs) and 95\% CI of newly diagnosed NAFLD were estimated using logistic regression models. We fitted five models in the analyses. The first model was unadjusted. The second model was adjusted for age, sex, and BMI. In model 3, we adjusted for smoking status, alcohol drinking status, educational level, occupation, household income, PA, family history of CVD, family history of hypertension, family history of hyperlipidemia, family history of diabetes, and intake of total energy, carbohydrate, and total fat, EPA and DHA, soft drinks, vegetables, fruits, sweet foods, and milk. In model 4, we additionally adjusted for hypertension, diabetes, and hyperlipidemia. In a final model, we further adjusted for WBC count $\left(\times 1000\right.$ cells $/ \mathrm{mm}^{3}$ : continuous). The linear trend was tested by assigning yogurt consumption category as an ordinal variable in the logistic models.

Interactions between yogurt consumption and potential confounders were examined by including the quadratic terms in the final model (model 5). Multicollinearity among covariates was tested by the variance inflation factor (VIF). Moreover, we conducted a sensitivity analysis after excluding participants with MS from the analysis. Data analysis was performed using SAS 9.3 software (SAS Institute Inc., Cary, NC). All statistical tests were two-sided, and $P<0.05$ was considered statistically significant.

\section{Results}

Baseline characteristics of the study population by yogurt consumption categories are shown in Table 1. Participants with more frequent consumption of yogurt tended to be younger, were women, had lower BMI, WC, TC, TG, LDLC, SBP, DBP, FBG level, and WBC count, but had higher HDL-C level ( $P$ for all trend $<0.0001$ ), and were more likely to exercise $(P$ for trend $<0.001)$. They also consumed more total energy, carbohydrate intake, total fat, vegetables, fruits, sweet foods, EPA and DHA, and soft drinks ( $P$ for all trend $<0.0001)$. In addition, participants with higher yogurt consumption were less likely to be current smokers, exsmokers, everyday drinkers, sometime drinkers, ex-drinkers, employed as professionals or other, and to have MS, hypertension, hyperlipidemia, diabetes, or a family history of CVD ( $P$ for all trend $<0.05$ ). Participants with higher yogurt consumption were more likely to be non-smokers, non-drinkers, employed as managers, and to have a family history of hyperlipidemia or diabetes $(P$ for all trend $<0.05)$. 
Table 1 Participant characteristics according to frequency of yogurt consumption $(n=24,389)$

\begin{tabular}{|c|c|c|c|c|c|}
\hline \multirow[t]{2}{*}{ Characteristics } & \multicolumn{4}{|l|}{ Yogurt consumption } & \multirow[t]{2}{*}{$P$ for trend ${ }^{\mathrm{a}}$} \\
\hline & $<1$ time/week & 1 time/week & 2-3 times/week & $\geq 4$ times/week & \\
\hline No. of subjects & 10,271 & 3389 & 5959 & 4770 & - \\
\hline Age (years) & $41.7(41.4,41.9)^{\mathrm{b}}$ & $37.0(36.7,37.4)$ & $36.8(36.5,37.0)$ & $37.7(37.4,38.0)$ & $<0.0001$ \\
\hline Men (\%) & 56.5 & 49.3 & 38.5 & 35.4 & $<0.0001$ \\
\hline BMI $\left(\mathrm{kg} / \mathrm{m}^{2}\right)$ & $24.1(24.1,24.2)$ & $23.5(23.4,23.6)$ & $23.3(23.2,23.4)$ & $23.3(23.2,23.4)$ & $<0.0001$ \\
\hline $\mathrm{WC}(\mathrm{cm})$ & $82.3(82.1,82.5)$ & $79.8(79.4,80.2)$ & $78.5(78.3,78.8)$ & $78.4(78.1,78.7)$ & $<0.0001$ \\
\hline $\mathrm{TC}(\mathrm{mmol} / \mathrm{L})$ & $4.72(4.71,4.74)$ & $4.60(4.57,4.63)$ & $4.60(4.58,4.62)$ & $4.62(4.59,4.64)$ & $<0.0001$ \\
\hline TG $(\mathrm{mmol} / \mathrm{L})$ & $1.15(1.13,1.16)$ & $1.02(1.00,1.03)$ & $0.98(0.97,0.99)$ & $0.97(0.95,0.98)$ & $<0.0001$ \\
\hline LDL-C $(\mathrm{mmol} / \mathrm{L})$ & $2.74(2.72,2.75)$ & $2.65(2.62,2.67)$ & $2.63(2.61,2.65)$ & $2.63(2.61,2.65)$ & $<0.0001$ \\
\hline HDL-C $(\mathrm{mmol} / \mathrm{L})$ & $1.32(1.31,1.33)$ & $1.36(1.35,1.37)$ & $1.39(1.38,1.40)$ & $1.41(1.40,1.42)$ & $<0.0001$ \\
\hline SBP (mmHg) & $120.3(120.0,120.6)$ & $117.1(116.6,117.7)$ & $116.3(115.9,116.7)$ & $116.8(116.4,117.2)$ & $<0.0001$ \\
\hline DBP $(\mathrm{mmHg})$ & $76.0(75.8,76.2)$ & $73.6(73.3,74.0)$ & $73.0(72.8,73.3)$ & $73.2(72.9,73.5)$ & $<0.0001$ \\
\hline FBG $(\mathrm{mmol} / \mathrm{L})$ & $5.05(5.04,5.07)$ & $4.90(4.87,4.92)$ & $4.89(4.87,4.91)$ & $4.88(4.86,4.90)$ & $<0.0001$ \\
\hline WBC count $\left(\times 1000\right.$ cells $\left./ \mathrm{mm}^{3}\right)$ & $5.72(5.70,5.75)$ & $5.69(5.65,5.74)$ & $5.62(5.59,5.66)$ & $5.61(5.57,5.65)$ & $<0.0001$ \\
\hline Physical activity (MET × hour/week) & $9.60(9.40,9.90)$ & $9.30(8.90,9.70)$ & $10.4(10.1,10.8)$ & $11.2(10.8,11.6)$ & $<0.001$ \\
\hline Total energy intake (kcal/day) & $1869.9(1859.4,1880.4)$ & $1961.8(1942.7,1981.1)$ & $2058.2(2043.0,2073.4)$ & $2180.1(2162.2,2198.1)$ & $<0.0001$ \\
\hline Carbohydrate intake (g/day) & $326.4(323.7,329.1)$ & $344.1(339.2,349.0)$ & $369.4(365.5,373.5)$ & $425.2(420.1,430.4)$ & $<0.0001$ \\
\hline Total fat intake (g/day) & $39.9(39.5,40.2)$ & $44.5(43.8,45.2)$ & $47.3(46.7,47.9)$ & $54.7(54.0,55.4)$ & $<0.0001$ \\
\hline Total vegetables intake (g/day) & $223.8(221.1,226.5)$ & $234.0(229.1,239.0)$ & $250.2(246.2,254.2)$ & $288.9(283.8,294.1)$ & $<0.0001$ \\
\hline Total fruits intake (g/day) & $208.1(203.8,212.6)$ & $271.8(262.0,281.9)$ & $310.4(301.9,319.1)$ & $351.5(340.8,362.6)$ & $<0.0001$ \\
\hline Sweet foods intake (g/day) & $11.4(11.1,11.8)$ & $21.9(20.8,23.1)$ & $23.7(22.8,24.7)$ & $23.1(22.1,24.1)$ & $<0.0001$ \\
\hline EPA and DHA intake (g/day) & $3.53(3.48,3.57)$ & $4.06(3.97,4.14)$ & $4.15(4.08,4.22)$ & $4.37(4.29,4.45)$ & $<0.0001$ \\
\hline Soft drinks intake (mL/day) & $4.00(3.85,4.15)$ & $6.71(6.29,7.15)$ & $5.94(5.66,6.24)$ & $4.91(4.65,5.18)$ & $<0.0001$ \\
\hline \multicolumn{6}{|l|}{ Smoking status (\%) } \\
\hline Smoker & 23.8 & 17.0 & 12.8 & 10.8 & $<0.0001$ \\
\hline Ex-smoker & 6.37 & 3.81 & 2.83 & 3.31 & $<0.0001$ \\
\hline Non-smoker & 69.8 & 79.2 & 84.4 & 85.9 & $<0.0001$ \\
\hline \multicolumn{6}{|l|}{ Alcohol drinking status (\%) } \\
\hline Everyday & 5.27 & 2.79 & 1.62 & 2.05 & $<0.0001$ \\
\hline Sometime & 55.6 & 56.6 & 54.7 & 52.0 & $<0.0001$ \\
\hline Ex-drinker & 10.8 & 10.3 & 9.81 & 9.24 & $<0.01$ \\
\hline Non-drinker & 28.3 & 30.3 & 33.9 & 36.7 & $<0.0001$ \\
\hline Education level (college or higher, \%) & 56.7 & 73.9 & 73.6 & 71.8 & $<0.0001$ \\
\hline \multicolumn{6}{|l|}{ Working status (\%) } \\
\hline Managers & 36.9 & 43.9 & 44.2 & 47.9 & $<0.0001$ \\
\hline Professionals & 17.5 & 18.3 & 17.0 & 13.6 & $<0.0001$ \\
\hline Other & 45.7 & 37.9 & 38.8 & 38.6 & $<0.0001$ \\
\hline $\begin{array}{l}\text { Household income ( } \geq 10,000 \\
\text { Yuan, \%) }\end{array}$ & 32.9 & 36.1 & 37.6 & 34.0 & $<0.01$ \\
\hline Metabolic syndrome (\%) & 25.5 & 16.6 & 16.6 & 15.6 & $<0.0001$ \\
\hline Hypertension (\%) & 25.3 & 16.1 & 14.3 & 15.3 & $<0.0001$ \\
\hline Hyperlipidemia (\%) & 44.3 & 36.0 & 34.7 & 36.0 & $<0.0001$ \\
\hline Diabetes $(\%)$ & 3.89 & 1.80 & 1.73 & 1.59 & $<0.0001$ \\
\hline \multicolumn{6}{|l|}{ Family history of disease $(\%)$} \\
\hline CVD & 27.7 & 25.7 & 25.8 & 26.7 & 0.049 \\
\hline Hypertension & 47.8 & 46.3 & 46.6 & 47.2 & 0.29 \\
\hline Hyperlipidemia & 0.11 & 0.30 & 0.32 & 0.31 & $<0.01$ \\
\hline Diabetes & 22.8 & 24.3 & 25.0 & 24.1 & 0.01 \\
\hline
\end{tabular}

$B M I$ body mass index, $W C$ waist circumference, $T C$ total cholesterol, $T G$ triglycerides, $L D L-C$ low-density lipoprotein cholesterol, $H D L-C$ highdensity lipoprotein cholesterol, $S B P$ systolic blood pressure, $D B P$ diastolic blood pressure, $F B G$ fasting blood glucose, $W B C$ white blood cell, $M E T$ metabolic equivalent, EPA eicosapentaenoic acid, DHA docosahexaenoic acid, $C V D$ cardiovascular disease

${ }^{\mathrm{a}}$ Analysis of variance or logistic regression analysis

${ }^{\mathrm{b}}$ Geometric mean (95\% confidence interval) (all such values)

As shown in Table 2, those with NAFLD tended to be older and were more likely to have a higher BMI, WC, TC,
TG, LDL-C, SBP, DBP, FBG, PA, and WBC count, but a lower HDL-C (all $P$ values $<0.0001$ ). In addition, those 
Table 2 Participant characteristics according to NAFLD status $(n=24,389)$

\begin{tabular}{|c|c|c|c|}
\hline \multirow[t]{2}{*}{ Characteristics } & \multicolumn{2}{|l|}{ NAFLD status } & \multirow[t]{2}{*}{$P$ value $^{\mathrm{a}}$} \\
\hline & No & Yes & \\
\hline No. of subjects & 19,731 & 4658 & - \\
\hline Age (years) & $38.3(38.2,38.5)^{\mathrm{b}}$ & $42.0(41.7,42.4)$ & $<0.0001$ \\
\hline Men (\%) & 41.2 & 71.6 & $<0.0001$ \\
\hline BMI $\left(\mathrm{kg} / \mathrm{m}^{2}\right)$ & $22.8(22.8,22.8)$ & $27.8(27.7,27.9)$ & $<0.0001$ \\
\hline WC (cm) & $77.6(77.4,77.7)$ & $92.7(92.4,93.0)$ & $<0.0001$ \\
\hline $\mathrm{TC}(\mathrm{mmol} / \mathrm{L})$ & $4.59(4.58,4.60)$ & $4.95(4.92,4.97)$ & $<0.0001$ \\
\hline TG $(\mathrm{mmol} / \mathrm{L})$ & $0.93(0.93,0.94)$ & $1.70(1.68,1.72)$ & $<0.0001$ \\
\hline LDL-C $(\mathrm{mmol} / \mathrm{L})$ & $2.62(2.60,2.63)$ & $2.93(2.91,2.96)$ & $<0.0001$ \\
\hline HDL-C $(\mathrm{mmol} / \mathrm{L})$ & $1.43(1.42,1.43)$ & $1.12(1.11,1.13)$ & $<0.0001$ \\
\hline SBP (mmHg) & $115.9(115.7,116.1)$ & $128.7(128.3,129.2)$ & $<0.0001$ \\
\hline $\mathrm{DBP}(\mathrm{mmHg})$ & $72.8(72.7,72.9)$ & $81.5(81.1,81.8)$ & $<0.0001$ \\
\hline $\mathrm{FBG}(\mathrm{mmol} / \mathrm{L})$ & $4.85(4.84,4.86)$ & $5.43(5.41,5.46)$ & $<0.0001$ \\
\hline WBC count $\left(\times 1000\right.$ cells $\left./ \mathrm{mm}^{3}\right)$ & $5.51(5.49,5.53)$ & $6.41(6.37,6.46)$ & $<0.0001$ \\
\hline Physical activity $($ MET $\times$ h/week) & $9.90(9.70,10.1)$ & $11.0(10.5,11.4)$ & $<0.0001$ \\
\hline Total energy intake (kcal/day) & $1985.9(1977.7,1994.2)$ & $1985.0(1968.2,2002.0)$ & 0.92 \\
\hline Carbohydrate intake (g/day) & $357.1(354.9,359.3)$ & $356.0(351.5,360.5)$ & 0.66 \\
\hline Total fat intake (g/day) & $44.6(44.3,44.9)$ & $46.1(45.5,46.8)$ & $<0.0001$ \\
\hline Total vegetables intake (g/day) & $243.6(241.4,245.8)$ & $241.8(237.4,246.2)$ & 0.47 \\
\hline Total fruits intake (g/day) & $267.2(263.1,271.4)$ & $250.2(242.3,258.3)$ & $<0.001$ \\
\hline Sweet foods intake (g/day) & $18.1(17.7,18.5)$ & $13.7(13.1,14.3)$ & $<0.0001$ \\
\hline EPA and DHA intake (g/day) & $3.87(3.84,3.91)$ & $4.02(3.95,4.10)$ & $<0.001$ \\
\hline Soft drinks intake (mL/day) & $4.83(4.70,4.96)$ & $5.37(5.08,5.67)$ & $<0.001$ \\
\hline \multicolumn{4}{|l|}{ Smoking status (\%) } \\
\hline Smoker & 15.2 & 28.3 & $<0.0001$ \\
\hline Ex-smoker & 4.01 & 6.88 & $<0.0001$ \\
\hline Non-smoker & 80.8 & 64.8 & $<0.0001$ \\
\hline \multicolumn{4}{|l|}{ Alcohol drinking status (\%) } \\
\hline Everyday & 3.71 & 2.09 & $<0.0001$ \\
\hline Sometime & 53.1 & 62.1 & $<0.0001$ \\
\hline Ex-drinker & 9.78 & 11.8 & $<0.0001$ \\
\hline Non-drinker & 33.4 & 24.0 & $<0.0001$ \\
\hline Education level (college or higher, \%) & 68.1 & 57.9 & $<0.0001$ \\
\hline \multicolumn{4}{|l|}{ Working status (\%) } \\
\hline Managers & 42.6 & 38.3 & $<0.0001$ \\
\hline Professionals & 16.6 & 17.1 & 0.37 \\
\hline Other & 40.8 & 44.6 & $<0.0001$ \\
\hline Household income ( $\geq 10,000$ Yuan, \%) & 35.0 & 33.8 & 0.13 \\
\hline Metabolic syndrome $(\%)$ & 11.2 & 55.5 & $<0.0001$ \\
\hline Hypertension $(\%)$ & 14.3 & 40.9 & $<0.0001$ \\
\hline Hyperlipidemia (\%) & 32.6 & 67.0 & $<0.0001$ \\
\hline Diabetes $(\%)$ & 1.31 & 8.18 & $<0.0001$ \\
\hline \multicolumn{4}{|l|}{ Family history of disease $(\%)$} \\
\hline CVD & 27.4 & 24.1 & $<0.0001$ \\
\hline Hypertension & 47.0 & 48.1 & 0.19 \\
\hline Hyperlipidemia & 0.27 & 0.02 & 0.01 \\
\hline Diabetes & 22.9 & 27.7 & $<0.0001$ \\
\hline
\end{tabular}

$B M I$ body mass index, $W C$ waist circumference, $T C$ total cholesterol, $T G$ triglycerides, $L D L-C$ low-density lipoprotein cholesterol, $H D L-C$ highdensity lipoprotein cholesterol, $S B P$ systolic blood pressure, $D B P$ diastolic blood pressure, $F B G$ fasting blood glucose, $W B C$ white blood cell, $M E T$ metabolic equivalent, $E P A$ eicosapentaenoic acid, $D H A$ docosahexaenoic acid, $C V D$ cardiovascular disease

${ }^{a}$ Analysis of variance or logistic regression analysis

${ }^{\mathrm{b}}$ Geometric mean (95\% confidence interval) (all such values)

with NAFLD had a higher intake of total fat, EPA and DHA, and soft drinks, and a lower intake of fruits, and sweet foods (all $P$ values $<0.001$ ). A higher proportion of these participants were men, current smokers, ex-smokers, sometime drinkers, ex-drinkers, employed as other, and had chronic conditions and family history of diabetes (all $P$ values 
Table 3 The association of yogurt consumption with NAFLD $(n=24,389)$

\begin{tabular}{|c|c|c|c|c|c|}
\hline \multirow[t]{2}{*}{ Logistic regression models } & \multicolumn{4}{|c|}{ Yogurt consumption } & \multirow[t]{2}{*}{$P$ for trend ${ }^{2}$} \\
\hline & $<1$ time/week & 1 time/week & 2-3 times/week & $\geq 4$ times/week & \\
\hline No. of subjects & 10,271 & 3389 & 5959 & 4770 & - \\
\hline No. of NAFLD & 2383 & 626 & 941 & 708 & - \\
\hline Model 1 & 1.00 (reference) & $0.75(0.68,0.83)^{\mathrm{b}}$ & $0.62(0.57,0.67)$ & $0.58(0.53,0.63)$ & $<0.0001$ \\
\hline Model 2 & 1.00 (reference) & $1.01(0.89,1.14)$ & $0.93(0.84,1.03)$ & $0.85(0.76,0.96)$ & $<0.01$ \\
\hline Model 3 & 1.00 (reference) & $0.97(0.85,1.10)$ & $0.89(0.80,0.99)$ & $0.84(0.74,0.95)$ & $<0.01$ \\
\hline Model 4 & 1.00 (reference) & $0.99(0.87,1.13)$ & $0.90(0.81,1.01)$ & $0.85(0.75,0.97)$ & $<0.01$ \\
\hline Model 5 & 1.00 (reference) & $1.00(0.88,1.14)$ & $0.91(0.81,1.02)$ & $0.86(0.76,0.98)$ & 0.01 \\
\hline
\end{tabular}

Model 1: crude model

Model 2: adjusted for age, sex, and body mass index

Model 3: model 2 plus further adjusted for smoking status, alcohol drinking status, education level, working status, household income, physical activity, family history of disease (including cardiovascular disease, hypertension, hyperlipidemia, and diabetes), total energy intake, carbohydrate intake, total fat intake, eicosapentaenoic acid + docosahexaenoic acid intake, soft drinks intake, vegetables intake, fruits intake, sweet foods intake, and milk intake

Model 4: model 3 plus further adjusted for hypertension, diabetes, and hyperlipidemia

Model 5: model 4 plus further adjusted for WBC count

NAFLD non-alcoholic fatty liver disease

${ }^{\mathrm{a}}$ Obtained by using multiple logistic regression analysis

${ }^{\mathrm{b}}$ Odds ratio (95\% confidence interval) (all such values)

$<0.0001)$. A lower proportion of these participants were nonsmokers, everyday drinkers, non-drinkers, had lower education level, were employed as managers, and had a family history of CVD or hyperlipidemia (all $P$ values $<0.05$ ).

Association between yogurt consumption and newly diagnosed NAFLD are presented in Table 3 . The unadjusted ORs $(95 \% \mathrm{CI})$ for newly diagnosed NAFLD comparing the 1 time/week, 2-3 times/week, and $\geq 4$ times/week of yogurt consumption as the $<1$ time/week were $0.75(0.68,0.83)$, $0.62(0.57,0.67)$, and $0.58(0.53,0.63)$ ( $P$ for trend $<$ 0.0001). After adjusting for age, sex, and BMI, the ORs (95\% CI) for NAFLD across increasing consumption categories of yogurt were 1.00 (reference), $1.01(0.89,1.14)$, $0.93(0.84,1.03)$, and $0.85(0.76,0.96)(P$ for trend $<0.01)$. Similar results were also observed after further adjustment for possible confounding and mediating factors (Table 3).

No significant interactions between yogurt consumption and covariates were observed in the fully adjusted model ( $P$ for interaction $>0.10$ ). There was no multicollinearity in the model 5 , as all VIF values were $<4.00$. In the sensitivity analysis, we excluded participants who had MS $(n=4520)$. However, results did not change substantially (data not shown).

\section{Discussion}

The main findings of the present study were that higher yogurt consumption was dose-dependently associated with a lower prevalence of newly diagnosed NAFLD. The largescale cross-sectional study is the first study performed to assess the association of yogurt consumption with NAFLD in a general population.

In this cross-sectional study, we included only newly diagnosed NAFLD, which reduced the potential influence of reverse causation on our analyses [18]. Moreover, we adjusted for many confounding factors. First, we adjusted for age, sex, and BMI because these factors were significantly different between groups. Adjustments for these factors substantially attenuated the observed association. This suggests that there is also the possibility of residual confounding since the subjects were quite different in terms of these important characteristics. In particular, BMI is a crude measure of body fat which is associated with NAFLD. However, data on body fat were not available for the present study. Therefore, imperfect measurement of body fat may cause a large bias in the estimated effects. Future studies should investigate the impact of yogurt consumption on NAFLD was independent of mediating by body fat. Second, we made adjustments for lifestyle factors and dietary intakes. However, there were similar results when we adjusted for smoking and alcohol drinking status, educational level, occupation, household income, PA, family medical history, total energy, carbohydrate, and total fat, EPA + DHA, soft drinks, vegetables, fruits, sweet foods, and milk intake. Thus, the observed association between yogurt consumption and newly diagnosed NAFLD was independent of these lifestyle and dietary factors. Third, 
yogurt consumption was associated with a lower risk of chronic diseases, which may contribute to a reduction of NAFLD [27, 28]. However, adjustments for hypertension, diabetes, and hyperlipidemia did not alter this inverse association. Furthermore, we excluded subjects with MS in a sensitivity analysis. However, a similar association was also observed. Therefore, yogurt consumption may be negatively associated with NAFLD independent of individual chronic diseases. Finally, we further adjusted for WBC count. After adjustment for the marker of subclinical inflammation, this inverse association was slightly attenuated and remained significant. This means that this inverse association was at least in part mediated through inflammation.

A double-blind RCT showed that probiotics (yogurt is one of the best sources) can improve liver aminotransferases levels in patients with NAFLD [29]. Accumulated evidence also indicated yogurt consumption was associated with improved metabolic profiles in humans, which might have preventive effects against NAFLD [30, 31]. Moreover, a double-blind 8-week RCT and an open-label 24-week RCT collectively showed that probiotic yogurt consumption could improve steatosis among NAFLD patients [14, 15]. However, to our knowledge, few studies have focused on the association of yogurt consumption and NAFLD in a general population. Therefore, our study filled this knowledge gap of association between yogurt consumption and NAFLD. These results need to be replicated in further investigations.

Although the exact mechanisms behind this inverse association remain unknown, these following several aspects may partly explain the observed results. First, yogurt is a rich source of probiotics. Animal experiments have suggested that probiotics may delay NAFLD development by suppressing the lipopolysaccharide and hepatic toll-like receptor 4 signaling pathway [32]. Second, previous studies have been proven that probiotics from yogurt to own anti-inflammatory, anti-oxidant, and immune-modulating activity, which might mediate the lower prevalence of NAFLD among individuals with higher yogurt consumption [33, 34]. Third, yogurt is one of the most nutrient-dense foods that are rich in proteins, minerals (e.g., calcium, magnesium, potassium), and vitamins. Evidence has shown that a higher intake of calcium contained in yogurt is associated with increased whole-body fat oxidation $[35,36]$. Thus, the inverse association might partly be explained by increased whole-body fat oxidation rates. Fourth, an animal study found that calcium and vitamin D combinations could prevent NAFLD development [37]. Finally, yogurt is seen as a nutrient-dense food linked with healthy diet habitats. Therefore, this association was partly explained by a healthier diet.

This study has several important advantages including the large sample size, and extensive information on lifestyle and dietary factors, which allowed us to control for many potential confounders. Several limitations of this study should also be noted. First, dietary information was selfreported. Thus, recall bias exists and the information on dietary intake may not be exact. Second, fatty liver disease was diagnosed by abdominal ultrasound rather than the gold standard liver biopsy. However, liver ultrasonography scanning is wildly used in large-scale population-based studies due to its non-invasiveness, easy accessibility, and high sensitivity and specificity [38]. Third, although we controlled for many confounders, we cannot rule out imprecision in the measurement of confounding factors included or residual confounding [39]. For example, varieties of PA and undocumented dietary factors could confound the observed associations. Thus, further confirmatory RCTs are needed to confirm the present findings. Finally, different yogurt types (such as whole fat or low-fat yogurt) may have a differential effect on NAFLD. However, low-fat yogurt was generally less common in China. Therefore, the effect of yogurt types on our results may be ignorable.

\section{Conclusions}

Our study results indicated that higher consumption of yogurt is associated with the reduced prevalence of NAFLD among Chinese adults. These results suggest that yogurt consumption may potentially contribute to the reduction of NAFLD. Future longitudinal studies are warranted to confirm this finding.

Acknowledgements We are grateful to all the people that have made this study. This study was supported by grants from the National Natural Science Foundation of China (Nos. 81673166, 81372118, 81372467 , and 81302422), the key technologies R\&D program of Tianjin (Key Project: Nos. 11ZCGYSY05700, 12ZCZDSY20400, 13ZCZDSY20200, and 15YFYZSY00020), the National Science and Technology Support Program (No. 2012BAI02B02), 2012 and 2016 Chinese Nutrition Society (CNS) Nutrition Research FoundationDSM Research Fund (Nos. 2014-071, 2016-046 and 2016-023), the Technologies development program of Beichen District of Tianjin (Nos. bcws2013-21, bcws2014-05 and 2015-SHGY-02), the technologies project of Tianjin Binhai New Area (Nos. 2013-02-04 and 2013-02-06), the Science Foundation of Tianjin Medical University (Nos. 2010KY28 and 2013KYQ24), the Key Laboratory of Public Health Safety (Fudan University), Ministry of Education (No. GW2014-5), and the National Training Programs of Innovation and Entrepreneurship for Undergraduates (No. 201510062013), China.

Author contributions SZ drafted the manuscript. JF, QZ, LL, ML, GM and $\mathrm{ZY}$ interpreted the results and contributed to the discussions. HW, $\mathrm{YX}, \mathrm{XB}, \mathrm{YG}, \mathrm{SS}, \mathrm{XW}, \mathrm{MZ}, \mathrm{QJ}, \mathrm{KS}, \mathrm{YW}$, and $\mathrm{HX}$ contributed to collect the data. $\mathrm{KN}$ designed the study, analyzed the data, reviewed, and edited the manuscript. KN had full access to all the data and took responsibility for the integrity of the data. All authors approved the final version. 


\section{Compliance with ethical standards}

Conflict of interest The authors declare that they have no conflict of interest.

Publisher's note: Springer Nature remains neutral with regard to jurisdictional claims in published maps and institutional affiliations.

\section{References}

1. Younossi ZM, Koenig AB, Abdelatif D, Fazel Y, Henry L, Wymer M. Global epidemiology of nonalcoholic fatty liver disease-Meta-analytic assessment of prevalence, incidence, and outcomes. Hepatology. 2016;64:73-84. https://doi.org/10.1002/ hep.28431.

2. Li Z, Xue J, Chen P, Chen L, Yan S, Liu L. Prevalence of nonalcoholic fatty liver disease in mainland of China: a meta-analysis of published studies. J Gastroenterol Hepatol. 2014;29:42-51. https://doi.org/10.1111/jgh.12428.

3. Targher G, Byrne CD, Lonardo A, Zoppini G, Barbui C. Nonalcoholic fatty liver disease and risk of incident cardiovascular disease: a meta-analysis. J Hepatol. 2016;65:589-600. https://doi. org/10.1016/j.jhep.2016.05.013.

4. Francque SM, van der Graaff D, Kwanten WJ. Non-alcoholic fatty liver disease and cardiovascular risk: Pathophysiological mechanisms and implications. J Hepatol. 2016;65:425-43. https:// doi.org/10.1016/j.jhep.2016.04.005.

5. Chalasani N, Younossi Z, Lavine JE, Charlton M, Cusi K, Rinella $\mathrm{M}$, et al. The diagnosis and management of nonalcoholic fatty liver disease: practice guidance from the American Association for the Study of Liver Diseases. Hepatology. 2018;67:328-57. https:// doi.org/10.1002/hep.29367.

6. Wen L, Duffy A. Factors influencing the gut microbiota, inflammation, and type 2 diabetes. J Nutr. 2017;147:1468S-75S. https:// doi.org/10.3945/jn.116.240754.

7. Ye H, Li Q, Zhang Z, Sun M, Zhao C, Zhang T. Effect of a novel potential probiotic Lactobacillus paracasei Jlus66 isolated from fermented milk on nonalcoholic fatty liver in rats. Food Funct. 2017;8:4539-46. https://doi.org/10.1039/c7fo01108c.

8. Xie N, Cui Y, Yin YN, Zhao X, Yang JW, Wang ZG, et al. Effects of two Lactobacillus strains on lipid metabolism and intestinal microflora in rats fed a high-cholesterol diet. BMC Complement Alter Med. 2011;11:53 https://doi.org/10.1186/ 1472-6882-11-53.

9. Esposito E, Iacono A, Bianco G, Autore G, Cuzzocrea S, Vajro P, et al. Probiotics reduce the inflammatory response induced by a high-fat diet in the liver of young rats. J Nutr. 2009;139:905-11. https://doi.org/10.3945/jn.108.101808.

10. Mouzaki M, Comelli EM, Arendt BM, Bonengel J, Fung SK, Fischer SE, et al. Intestinal microbiota in patients with nonalcoholic fatty liver disease. Hepatology. 2013;58:120-7. https://doi. org/10.1002/hep.26319.

11. Ma X, Hua J, Li Z. Probiotics improve high fat diet-induced hepatic steatosis and insulin resistance by increasing hepatic NKT cells. J Hepatol. 2008;49:821-30. https://doi.org/10.1016/j. jhep.2008.05.025.

12. Kim YA, Keogh JB, Clifton PM. Probiotics, prebiotics, synbiotics and insulin sensitivity. Nutr Res Rev. 2018;31:35-51. https://doi. org/10.1017/S095442241700018X.

13. Pereira DI, Gibson GR. Effects of consumption of probiotics and prebiotics on serum lipid levels in humans. Crit Rev Biochem Mol Biol. 2002;37:259-81. https://doi.org/10.1080/10409230290771519.
14. Bakhshimoghaddam F, Shateri K, Sina M, Hashemian M, Alizadeh M. Daily consumption of synbiotic yogurt decreases liver steatosis in patients with nonalcoholic fatty liver disease: a randomized controlled clinical trial. J Nutr. 2018;148:1276-84. https://doi.org/10.1093/jn/nxy088.

15. Nabavi S, Rafraf M, Somi MH, Homayouni-Rad A, AsghariJafarabadi M. Effects of probiotic yogurt consumption on metabolic factors in individuals with nonalcoholic fatty liver disease. $\mathbf{J}$ Dairy Sci. 2014;97:7386-93. https://doi.org/10.3168/jds.20148500.

16. Farrell GC, Chitturi S, Lau GK, Sollano JD. Asia-Pacific Working Party on NAFLD. Guidelines for the assessment and management of non-alcoholic fatty liver disease in the Asia-Pacific region: executive summary. J Gastroenterol Hepatol. 2007;22:775-7. https://doi.org/10.1111/j.1440-1746.2007.05002.x.

17. Zeng MD, Fan JG, Lu LG, Li YM, Chen CW, Wang BY, et al. Guidelines for the diagnosis and treatment of nonalcoholic fatty liver diseases. J Dig Dis. 2008;9:108-12. https://doi.org/10.1111/ j.1751-2980.2008.00331.x.

18. Zhang S, Fu J, Zhang Q, Liu L, Meng G, Yao Z, et al. Association between nut consumption and non-alcoholic fatty liver disease in adults. Liver Int. 2019. https://doi.org/10.1111/liv.14164.

19. Yang YX, Wang GY, Pan XC. China food composition. 2nd ed. Beijing: Peking University Medical Press; 2009.

20. Yu B, Zhu Q, Meng G, Gu Y, Zhang Q, Liu L, et al. Habitual yoghurt consumption and depressive symptoms in a general population study of 19,596 adults. Eur J Nutr. 2018;57:2621-8. https://doi.org/10.1007/s00394-017-1532-x.

21. Craig CL, Marshall AL, Sjostrom M, Bauman AE, Booth ML, Ainsworth BE, et al. International physical activity questionnaire: 12-country reliability and validity. Med Sci Sports Exerc. 2003;35:1381-95. https://doi.org/10.1249/01.MSS.0000078924. 61453.FB.

22. Mancia G, De Backer G, Dominiczak A, Cifkova R, Fagard R, Germano G, et al. 2007 Guidelines for the Management of Arterial Hypertension: The Task Force for the Management of Arterial Hypertension of the European Society of Hypertension (ESH) and of the European Society of Cardiology (ESC). J Hypertens. 2007;25:1105-87. https://doi.org/10.1097/HJH.0b013e3281fc975a.

23. Chobanian AV, Bakris GL, Black HR, Cushman WC, Green LA, Izzo JL, Jr. et al. The Seventh Report of the Joint National Committee on Prevention, Detection, Evaluation, and Treatment of High Blood Pressure: the JNC 7 report. JAMA. 2003;289:2560-72. https://doi.org/10.1001/jama.289.19.2560.

24. Alberti KG, Zimmet PZ. Definition, diagnosis and classification of diabetes mellitus and its complications. Part 1: diagnosis and classification of diabetes mellitus provisional report of a WHO consultation. Diabet Med. 1998;15:539-53.

25. Alberti KG, Eckel RH, Grundy SM, Zimmet PZ, Cleeman JI, Donato KA, et al. Harmonizing the metabolic syndrome: a joint interim statement of the International Diabetes Federation Task Force on Epidemiology and Prevention; National Heart, Lung, and Blood Institute; American Heart Association; World Heart Federation; International Atherosclerosis Society; and International Association for the Study of Obesity. Circulation. 2009;120:1640-5. https://doi.org/10.1161/CIRCULATIONAHA. 109.192644.

26. Gu Y, Hu K, Huang Y, Zhang Q, Liu L, Meng G, et al. White blood cells count as an indicator to identify whether obesity leads to increased risk of type 2 diabetes. Diabetes Res Clin Pract. 2018;141:140-7. https://doi.org/10.1016/j.diabres.2018.04.041.

27. Buendia JR, Li Y, Hu FB, Cabral HJ, Bradlee ML, Quatromoni $\mathrm{PA}$, et al. Regular yogurt intake and risk of cardiovascular disease among hypertensive adults. Am J Hypertens. 2018;31:557-65. https://doi.org/10.1093/ajh/hpx220. 
28. Chen M, Sun Q, Giovannucci E, Mozaffarian D, Manson JE, Willett WC, et al. Dairy consumption and risk of type 2 diabetes: 3 cohorts of US adults and an updated meta-analysis. BMC Med. 2014;12:215. https://doi.org/10.1186/s12916-014-0215-1.

29. Aller R, De Luis DA, Izaola O, Conde R, Gonzalez Sagrado M, Primo D, et al. Effect of a probiotic on liver aminotransferases in nonalcoholic fatty liver disease patients: a double blind randomized clinical trial. Eur Rev Med Pharm Sci. 2011;15:1090-5.

30. Wang H, Livingston KA, Fox CS, Meigs JB, Jacques PF. Yogurt consumption is associated with better diet quality and metabolic profile in American men and women. Nutr Res. 2013;33:18-26. https://doi.org/10.1016/j.nutres.2012.11.009.

31. Hobbs DA, Givens DI, Lovegrove JA. Yogurt consumption is associated with higher nutrient intake, diet quality and favourable metabolic profile in children: a cross-sectional analysis using data from years 1-4 of the National diet and Nutrition Survey, UK. Eur J Nutr. 2019;58:409-22. https://doi.org/10.1007/s00394-017-1605-x.

32. Xue L, He J, Gao N, Lu X, Li M, Wu X, et al. Probiotics may delay the progression of nonalcoholic fatty liver disease by restoring the gut microbiota structure and improving intestinal endotoxemia. Sci Rep. 2017;7:45176. https://doi.org/10.1038/ srep45176.

33. Maddur H, Neuschwander-Tetri BA. More evidence that probiotics may have a role in treating fatty liver disease. Am J Clin Nutr. 2014;99:425-6. https://doi.org/10.3945/ajcn.113.082636.
34. Eslamparast T, Poustchi H, Zamani F, Sharafkhah M, Malekzadeh R, Hekmatdoost A. Synbiotic supplementation in nonalcoholic fatty liver disease: a randomized, double-blind, placebo-controlled pilot study. Am J Clin Nutr. 2014;99:535-42. https://doi.org/10. 3945/ajcn.113.068890.

35. Melanson EL, Sharp TA, Schneider J, Donahoo WT, Grunwald GK, Hill JO. Relation between calcium intake and fat oxidation in adult humans. Int J Obes Relat Metab Disord. 2003;27:196-203. https://doi.org/10.1038/sj.ijo.802202.

36. Zemel MB. Proposed role of calcium and dairy food components in weight management and metabolic health. Phys Sportsmed. 2009;37:29-39. https://doi.org/10.3810/psm.2009.06.1707.

37. Shojaei Zarghani S, Soraya H, Alizadeh M. Calcium and vitamin $\mathrm{D}_{3}$ combinations improve fatty liver disease through AMPKindependent mechanisms. Eur J Nutr. 2018;57:731-40. https://doi. org/10.1007/s00394-016-1360-4.

38. Amarapurkar DN, Hashimoto E, Lesmana LA, Sollano JD, Chen $\mathrm{PJ}$, Goh KL, et al. How common is non-alcoholic fatty liver disease in the Asia-Pacific region and are there local differences? J Gastroenterol Hepatol. 2007;22:788-93. https://doi.org/10.1111/j. 1440-1746.2007.05042.x.

39. Agoritsas T, Merglen A, Shah ND, O'Donnell M, Guyatt GH. Adjusted analyses in studies addressing therapy and harm: users' guides to the medical literature. JAMA. 2017;317:748-59. https:// doi.org/10.1001/jama.2016.20029. 